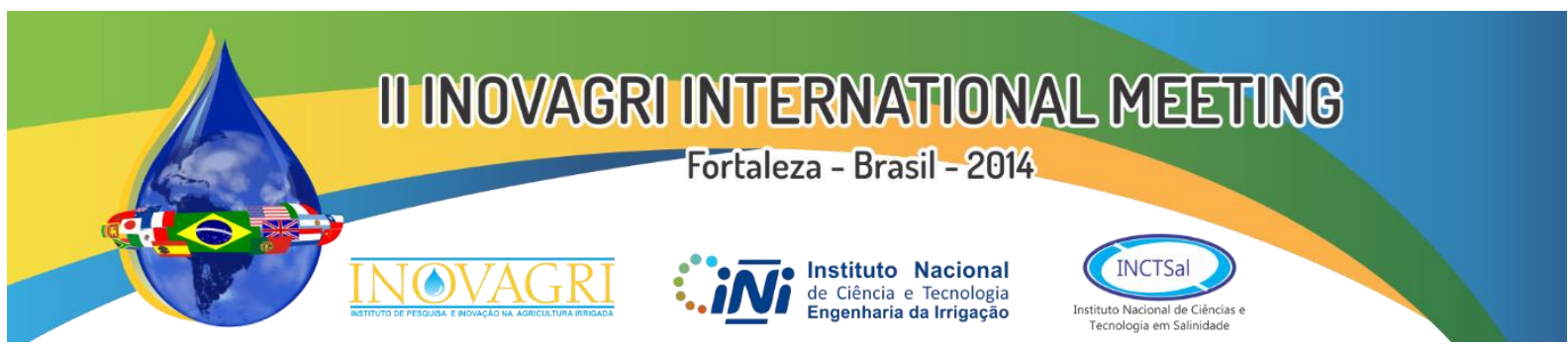

http://dx.doi.org/10.12702/ii.inovagri.2014-a151

\title{
UTILIZAÇÃO DO MODELO ZAE/FAO PARA A ESTIMATIVA DA PRODUTIVIDADE DO SORGO EM ALAGOAS
}

\author{
A. C. BARROS ${ }^{1}$, R. F. de SOUZA' 2 , A. H. C. BARROS ${ }^{3}$, J. N. TABOSA ${ }^{4}$, A. W. A. GOMES 5 , K. N. \\ TAVARES ${ }^{6}$
}

RESUMO: O manejo adequado, como a escolha da época de plantio ou o uso da irrigação, permite o maior aproveitamento dos recursos hídricos disponíveis no ambiente. Pode-se estimar a produtividade agrícola da cultura do sorgo através de modelos, desde que calibrados, permitindo variar os manejos. Dessa forma é possível conhecer a resposta da cultura à disponibilidade hídrica de cada local e sua variação ao longo do ano. Assim o objetivo do trabalho foi calibrar o modelo ZAE, estimar a produtividade e avaliar quais as épocas de plantio com menor risco para a cultura do sorgo no Estado de Alagoas. Para calibração do modelo ZAE/FAO foram utilizados dados provenientes do Programa de Melhoramento Genético de Sorgo do Nordeste na região de Araripina - PE, após a calibração foram simulados os cenários para as cidades de Água Branca, Arapiraca, Palmeira dos Índios e Porto de Pedras, em Alagoas, baseados na época de plantio (a cada 10 dias) x CAD do solo (30, 40 e $65 \mathrm{~mm}$ ) X sistema de plantio (sequeiro). O modelo apresentou ótimo ajuste, com $\mathrm{R}^{2}=0,87$; índice de Wilmont $(\mathrm{d})=0,94$; índice de confiabilidade (IC) de 0,87. As produtividades variaram de 1,8 a 1,9 Mg ha- ${ }^{-1}$, em Água Branca, de 3,7 a 4,1 Mg ha- ${ }^{1}$ em Arapiraca, 1,9 a 2,0 Mg ha- ${ }^{1}$ em Palmeira dos Índios, 2,2 a 2,3 $\mathrm{Mg}$ ha- $^{-1}$ em Porto de Pedras, no mês de plantio mais produtivo.

PALAVRA-CHAVE: modelagem matemática, disponibilidade hídrica, ISNA

\section{USE OF MODEL ZAE / FAO FOR ESTIMATING PRODUCTIVITY OF SORGHUM IN ALAGOAS}

\begin{abstract}
The proper management, the choice of season planting or the use of irrigation, allows better use of available water resources in the environment. Is possible to estimate productivity of sorghum through models, since it is calibrated, allowing to change the managements. Thus it is possible to meet the crop response to availability water at each location and its variation over the year. The aim of this study was to calibrate the model ZAE, estimating productivity and assess which planting times with less risk to sorghum in the state of Alagoas. To calibrate the model ZAE / FAO data were used from the breeding program of sorghum in the Northeast, region of Araripina - PE, after calibration scenarios were simulated for the cities of Água Branca, Arapiraca, Palmeira dos Indios and Porto de Pedras in Alagoas, based on planting time (every 10 days) x CAD soil (30,40 and $65 \mathrm{~mm}$ ) x cropping system (rainfed), and estimated the Water Satisfaction Needs Index (ISNA) for the quantification of water availability site. The model shows a good fit, with R2 $=0.87$, index Wilmont $(\mathrm{d})=0.94$; reliability index $(\mathrm{CI})$ of 0.87 . Yields ranged from 1.8 to $1.9 \mathrm{Mg} \mathrm{ha}^{-1}$ in Água Branca, 3.7 to $4.1 \mathrm{Mg}$ ha- $^{1}$ in Arapiraca, 1.9 to $2.0 \mathrm{Mg}^{-1}{ }^{-1}$ in Palmeira dos Índios , 2.2 to $2.3 \mathrm{Mg}^{\text {ha- }}{ }^{-1}$ in Porto de Pedras.
\end{abstract}

KEYWORDS: mathematical modeling, water availability, ISNA

\footnotetext{
${ }^{1}$ Doutor, Prof. UFAL, Arapiraca - Alagoas. Email: allan.cunha.barros@gmail.com

${ }^{2}$ Mestrando, Agricultura e Ambiente, Arapiraca - Alagoas. Email:

${ }^{3}$ Doutor, Pesquisador da Embrapa Solos, Recife - Pernambuco. Email: alex.hugo.2006@gmail.com

${ }^{4}$ Doutor, Pesquisador do IPA, Recife - Pernambuco. Email: nildo.tabosa@ipa.br

${ }^{5}$ Pós-Dr., UNESP, Botucatu - SP. Email: awagomes@ hotmail.com

${ }^{6}$ Estudante de Agronomia Arapiraca - Alagoas. Email: klinger.nunes@gmail.com
} 


\section{INTRODUÇÃO}

O sorgo (Sorghum bicolor) é uma planta de origem tropical, bem adaptada a regiões áridas e semiáridas, exigindo clima quente para poder expressar o seu potencial produtivo. A cultura, com características xerófilas, é considerada tolerante a períodos secos, notadamente em regiões do Nordeste do Brasil. No Nordeste, o cultivo do sorgo é realizado durante a estação chuvosa, cujo o período é curto e com distribuição irregular, caracterizado, ainda, por ocorrência de veranicos, com 15 a 20 dias sem chuvas. Em Pernambuco, as principais regiões produtoras localizam-se no Agreste e no Sertão (TABOSA, 2012).

A cultura do sorgo exige em torno de $300 \mathrm{~mm}$ a $400 \mathrm{~mm}$ de precipitação pluviométrica, distribuídos regularmente durante o seu ciclo de crescimento e desenvolvimento para que se alcancem níveis de produtividade satisfatórios. As fases fenológicas críticas da cultura, quanto à deficiência hídrica, correspondem ao estádio de plântula e no florescimento, sendo importante nessas épocas um adequado nível de suprimento de água para uma boa produção (TABOSA et al. 2008). A temperatura ótima para o desenvolvimento da cultura varia conforme a cultivar considerada. De modo geral, temperaturas do ar superiores a $38{ }^{\circ} \mathrm{C}$ ou inferiores a $16{ }^{\circ} \mathrm{C}$ limitam o desenvolvimento da maioria das cultivares.

O sorgo apresenta elevado potencial para ser utilizado na alimentação de animais, sobretudo nas regiões semiáridas, devido às características de resistência (OLIVEIRA et al. 2002). Contudo, a produtividade média é considerada baixa no Nordeste, em torno de 3.128 $\mathrm{kg} \mathrm{ha}^{-1}$ (IBGE, 2013), principalmente devido à falta de manejo adequado, com relação a pouca aplicação de fertilizantes e a densidade de plantio inadequada e, além disso, a irregularidade espaço-temporal da precipitação pluviométrica (SANTOS et al. 2013).

Nesse sentido, a estimativa da produtividade para a cultura do sorgo, utilizando o modelo de Zona Agroecológica (ZAE) da FAO (Doorenbos e Kassam, 1979) pode ajudar no planejamento agrícola de localidades no Nordeste Brasileiro. O modelo de ZAE é um dos mais utilizados em estudos para estimar a produtividade agrícola de culturas (ASSAD et al. 2007; ANDRIOLLI e SENTELHAS, 2009).

Visando um melhor aproveitamento dos recursos hídricos e o conhecimento das melhores épocas de se produzir o sorgo em Alagoas, desenvolveu-se esse trabalho com o objetivo de calibrar o modelo ZAE, para estimar a produtividade e avaliar quais as épocas de plantio com menor risco para a cultura do sorgo no Estado de Alagoas.

\section{MATERIAL E MÉTODOS}

O estudo foi desenvolvimento na Universidade Federal de Alagoas, Campus Arapiraca em parceria com o Instituto Agronômico de Pernambuco - IPA e a Embrapa Solos, UEP Recife.

O projeto foi divido em duas partes, na primeira foi feita a calibração do modelo para a cultura do sorgo, na segunda, foram feitas simulações de produtividade do sorgo para os municípios de Alagoas.

\section{Descrição do modelo}

O modelo de Zona Agroecológica (ZAE) estima a produtividade máxima da cultura, também chamada de produtividade potencial (PP) (DOORENBOS e KASSAM, 1979; BATTISTI, 2013), cujo os conceitos seguem os princípios de De Wit (1965). O modelo é calculado em duas etapas: a) estimativa da produtividade máxima ou potencial (Ymp) e b) a penalização da produtividade potencial, devido à deficiência hídrica da cultura, obtendo-se a produtividade real ou atingível (Yr). 


$$
\mathrm{Ymp}=\mathrm{cL} \times \mathrm{cN} \times \mathrm{cH} \times \mathrm{G}[\mathrm{F}(\mathrm{a}+\mathrm{b} \times \mathrm{ym}) \mathrm{yo}+(1-\mathrm{F})(\mathrm{c}+\mathrm{d} \times \mathrm{ym}) \mathrm{yc}]
$$

Em que:

Ymp - Produtividade potencial máxima da cultura (kg.ha- ${ }^{1}$.período-1); $\mathrm{cL}$ - correção em função do desenvolvimento da cultura e área foliar; $\mathrm{cN}$ - correção para a produção de matéria seca; $\mathrm{cH}$ correção para a parte colhida; G - período total do crescimento (dia); F - Fração do dia em que o sol fica encoberto por nuvens; Ym - Taxa de produção de matéria seca das folhas (kg.ha-1 ${ }^{1}$.período-1); a, b, c e d - Coeficientes da equação (tabelados); Yc - taxa de produção bruta de matéria seca $(\mathrm{kg} / \mathrm{ha} /$ perío kg.ha- ${ }^{1}$.período- ${ }^{1}$ do).

A produtividade real (Yr) é calculada por meio da correlação do crescimento da cultura com o consumo de água. O fator de sensibilidade devido ao déficit hídrico (Ky) é calculado de acordo com as fases fenológicas da cultura. Para a estimativa da produtividade real (Yr) o modelo ZAE utiliza a produtividade potencial máxima da cultura (Ymp), o coeficiente de resposta da cultura, o total da evapotranspiração real e o total da evapotranspiração máxima da cultura durante seu ciclo (Oliveira et al., 2010). Dessa forma, calcula-se o Yr da seguinte forma (Eq. 2):

$$
Y r=Y m p\left[1-k y\left(1-\frac{E T r}{E T p}\right)\right]
$$

em que, Ky é o coeficiente empírico, que representa a sensibilidade da cultura a deficiência hídrica (-); ETr é a evapotranspiração real da cultura $\left(\mathrm{mm} \mathrm{d}^{-1}\right)$; e ETp é a evapotranspiração máxima da cultura $\left(\mathrm{mm} \mathrm{d}^{-1}\right)$. O cálculo da ETp é realizado por meio da seguinte equação (3):

$$
E T p=E T o \times K c
$$

em que, ETp é a evapotranspiração máxima da cultura $\left(\mathrm{mm} \mathrm{d}^{-1}\right)$; ETo é a evapotranspiração de referência $\left(\mathrm{mm} \mathrm{d}^{-1}\right)$; e Kc o coeficiente de cultura. A ETo foi calculada de acordo com Penman-Moneith (ALLEN et al. 1998). Os valores de Kc utilizados nas fases fenológicas foram: fase I $(0,4)$; fase II $(1,1)$; fase III $(0,88)$; e fase IV $(0,58)$. Para a determinação da ETr utilizou-se o coeficiente de estresse hídrico (Ks) que penaliza o consumo de água da planta em função do déficit hídrico do solo (Eq. 4):

$$
K s=\frac{\ln [L A A+1]}{\ln [C T A+1]}
$$

em que, LAA é a lâmina de água no solo $(\mathrm{mm})$; CTA é a capacidade total de água no solo (mm). Assim, ETr é calculado por meio da equação:

$$
E T r=E T o \times K c \times K s
$$

\section{Calibração}

Para a calibração do modelo foram utilizados dados de produtividade do sorgo na região de Araripina - PE (lat. 07³4'34'S, long. 40²9'54'W, altitude de 622m), durante um período de quinze anos (1988 e 1899, 1991 a 2003), coletados do Programa de Melhoramento Genético de Sorgo do Nordeste da Embrapa/IPA. Os dados foram os mesmos utilizados por Barros (2010) e Martins (2012), que utilizaram modelos para prever a produtividade da cultura do sorgo nessas regiões.

O ciclo da cultura foi estabelecido em 129 dias, compreendendo quatro fases fenológicas: $1^{\text {a }}$ fase, 22 dias, da emergência até a diferenciação; $2^{\mathrm{a}}$ fase, 36 dias, reprodutiva; 
$3^{\text {a }}$ fase, 42 dias, do emborrachamento à floração; e a $4^{\text {a }}$ fase, 29 dias, da fase leitosa a maturação fisiológica (MAGALHÃES et al. 2000).

Os valores utilizados para as correções do modelo foram provenientes da literatura sobre a fisiologia da cultura do sorgo (EMBRAPA, 2013), os quais foram adaptados de acordo com as calibrações realizadas para a região de Araripina, PE. Adotou-se como profundidade do sistema radicular da cultura como $0,15 \mathrm{~m} ; 0,45 \mathrm{~m}$ e $0,5 \mathrm{~m}$, para as fases fenológicas I e II; III e IV, respectivamente.

Os dados de solo foram coletados de Barros (2010) e consistiu basicamente dos valores de Capacidade de Campo e Ponto de Murcha (à base volume); os dados climatológicos foram cedidos pelo Instituto Agronômico de Pernambuco - IP

As variáveis utilizadas na calibração foram a $\mathrm{cL}$ - correção em função do desenvolvimento da cultura e área foliar; $\mathrm{cN}$ - correção para a produção de matéria seca; $\mathrm{cH}$ correção para a parte colhida. Utilizou-se o procedimento de tentativa e erro por análise visual.

A avaliação do desempenho da estimativa da produtividade da cultura do sorgo foi avaliada utilizados os seguintes índices estatísticos: coeficiente de determinação $\left(\mathrm{R}^{2}\right)$, o índice de Willmott (d) e o índice de concordância (IC) de Camargo e Sentelhas (1997).

\section{Cenários simulados}

Como os modelos possuem como princípio básico a utilização em diversas regiões, e não se dispunha de dados de produtividade para a região de Alagoas, assim, considerou-se a calibração feita para Araripina-PE, como válida para a utilização nas simulações dos cenários em Alagoas. Ressaltando que nos dados tabelados do modelo ZAE não há diferença entre a latitude $0^{\circ}$ até $10^{\circ}$.

Os cenários da simulação foram baseados nas épocas de plantio e na disponibilidade de água para a cultura. Épocas de plantio (3 datas por mês - a cada 10 dias) X Disponibilidade de água (3 CAD - 65, 40 e $30 \mathrm{~mm}$ ), totalizando 108 cenários.

As cidades simuladas foram Arapiraca (lat. 0945'09'S, long. 36³9'40'W, altitude de

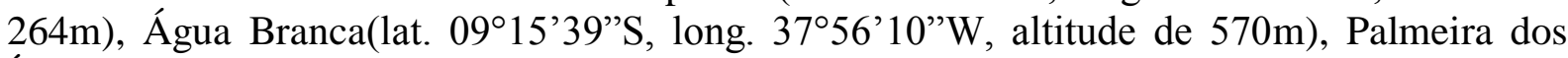
Índios (lat. 09²4'26”S, long. 36 37'39'W, altitude de 342m) e Porto de Pedras (lat. $09^{\circ} 09^{\prime} 30^{\prime}$ 'S, long. $35^{\circ} 17^{\prime} 42^{\prime \prime} \mathrm{W}$, altitude de $22 \mathrm{~m}$ ), cujos dados climatológicos foram coletados do Instituto Nacional de Meteorologia - IMET.

\section{RESULTADOS E DISCUSSÃO}

A diferença média entre a produtividade da cultura do sorgo observada e estimada é da ordem de $\pm 183 \mathrm{~kg} \mathrm{ha}^{-1}$, a mesma ordem de grandeza também é verificada entre os valores. A estimativa de produtividade superestimou os valores observados em $40 \%$ dos anos (6 anos) e subestimou em $60 \%$ (9 anos), com erro médio de $818 \mathrm{~kg} \mathrm{ha}^{-1}$. Apenas no ano de 1993 que a produtividade observada atingiu um valor muito inferior a media de $3.467 \mathrm{~kg} \mathrm{ha}^{-1}$ (Tabela 1). É provável que esta produtividade tenha ocorrido devido à ocorrência do efeito El Niño nesse ano (Martins, 2011).

O modelo da Zona Agroecológica utiliza parâmetros de cultura, como o coeficiente de sensibilidade a deficiência hídrica (Ky) e o coeficiente de colheita $(\mathrm{cH})$. Esses coeficientes são sensíveis a variações climáticas, principalmente a eventos extremos como a ocorrência de eventos El-Ninõ-Oscilação Sul (ENOS). Observa-se que os anos de fortes e moderados ElNiño (1992/1993, 1997/1998 e 2001/2002), a diferença entre os valores estimados e observados de produtividade tendem a serem maiores (TABELA 1).

O modelo ZAE foi testado em praticamente todas as regiões no Brasil, utilizando diversas culturas agrícolas, com resultados que auxiliam ao produtor minimizar as perdas de 
produtividade decorrentes do estresse hídrico (ROLIM et al. 2001; BONNECARRÈRE et al. 2007; ANDRIOLI e SENTELHAS, 2009; SHRESTHA et al., 2010).

Para a localidade de Araripina, PE o índice de Willmott foi de 0,93 e o de concordância (IC) 0,87 (ótimo), evidenciando o potencial do modelo ZAE para ser utilizado em diferentes estudos, devido à sua simplicidade, facilidade de ajuste e aplicabilidade (Figura 1). Verifica-se, ainda, pouca dispersão entre os dados de produtividade observados e estimados (reta 1:1) (Figura 1). Nesse sentido, quando se analisa a proporção do erro médio de $818 \mathrm{~kg} \mathrm{ha}^{-1}$ na estimativa da produtividade, por meio das barras de erros, observa-se que a maioria dos valores estimados estão dentro do intervalo permitido (Figura 2).

O índice estatístico (IC) demonstrou resultado ótimo próximo aos encontrados por Martin (2012) no município de Araripina, PE, utilizando os modelos de Zona Agroecológica e de Wageningen. Segundo Sentelhas (2011) vários aspectos devem ser considerados ao escolher o modelo de balanço hídrico para o monitoramento da umidade do solo, pois a principal limitação no uso de modelos mais complexos é o grande número e a complexidade das variáveis de entrada. De fato, muita vezes os dados necessários para uma avaliação mais completa não estão disponíveis, tornado o modelo ZAE uma ferramenta indispensável para avaliação de riscos na agricultura, principalmente em regiões, onde a variabilidade da precipitação pluviométrica é bastante acentuada, como a região do Nordeste do Brasil.

\section{Cenários Simulados}

Na Figura 3 estão as produtividades para os municípios simulados com a CAD de $65 \mathrm{~mm}$ (CAD 1), os outros solos (CAD $2-45 \mathrm{~mm}$ e CAD 3- $30 \mathrm{~mm}$ ) obtiveram a mesma tendência das curvas, por isso não houve necessidade de representá-las. As curvas de produtividade possuem uma similaridade com o regime hídrico local. Alves et al. (2011) relacionam esse acontecimento, redução da disponibilidade hídrica, com a queda de produtividade devido a redução do número de sementes na panícula.

As curvas das cidades de Arapiraca (ARA) e Palmeira dos Índios (PAI) possuem maior similaridade com as curvas obtidas por Marin et al (2006) para as regioes de Manduri, Piracicaba, Ribeirão Preto e Ilha Solteira, em São Paulo. Os municípios de Porto das Pedras (PDP) e Água Branca (AGB) possuem menor inclinação das curvas, demonstrando que ocorre menor variação de temperatura na região durante $\mathrm{O}$ ano.

Observa-se que a cidade de Arapiraca obteve as maiores produtividades, essa tem relação direta com a disponibilidade hídrica local e a região de PAI e AGB as menores, Assis et al. (2011) obtveram exatamente esse comportamento para a condição de sequeiro, onde a disponibilidade hídrica respondeu por $61,9 \%$ da variação total da produtividade, enquanto que os $38,1 \%$ restantes correspondem a fatores como data de plantio, ano de plantio, radiação solar e temperatura. As épocas de maior produtividade foram para os meses com plantio entre abril a junho, em ARA e PAI, de janeiro a junho em POP e para a cidade de AGB, os meses foram entre março a maio.

A variação da CAD, como já esperado, proporcionou redução das produtividades (TABELA 2), apesar da redução das capacidades de armazenamento de aproximadamente $38 \%$ da CAD 1 para a 2 , e de $53 \%$ para a 3 , as produtividades não acompanharam tão acentuadamente as reduções das CADs.

$\mathrm{Na}$ cidade de ARA as reduções ocorreram principalmente nos meses com menor disponibilidade hídrica, com início de produção em julho. Vale ressaltar que o período de plantio em julho irá sofrer déficit hídrico nos meses seguintes, principalmente, de setembro e outubro, época relacionada a fase de formação e enchimento dos grãos, uma vez que ocorre a redução na absorção de nutrientes e na área foliar, acarretando abaixamento na produção de fotoassimilados, (ALVES et al., 2011). A redução foi em média 10,0\% para a CAD 2 e 15,7\% para a CAD 3. As maiores reduções ocorreram para o mês de plantio em setembro em todas as cidades. 
As cidades de POP obtive menores reduções da produtividade em função da redução da CAD, em 7,8 e $12,2 \%$, CAD 2 e 3 . Pelo fato de PAI ser mais próximo a ARA, essa obteve semelhança entre o período de maior redução de produtividade.

Em AGB apresentou maiores reduções e o principal fator envolvido foi a baixa disponibilidade hídrica local, a cidade de AGB está localizada no sertão de Alagoas, apresentando precipitações médias anuais abaixo de $450 \mathrm{~mm}$ (CPRM, 2005) mesmo que o sorgo necessite de menos água para conversão em matéria seca, quando comparado ao milho e ao trigo, essas precipitações muitas das vezes nas regiões sertanejas ocorrem em um período considerado curto ao ciclo da cultura, representando déficit hídrico como no florescimento, fase crítica da cultura. (MAGALHÃES et al, 2000; A média de redução da produtividade na CAD 2 foi de 11,8\% e de 18,1\% pra a CAD 2.

\section{CONCLUSÕES}

A calibração do modelo da Zona Agroecológica - ZAE apresentou bons índices estatísticos podendo ser utilizado na simulação do sorgo com IC de 0,87 ;

As produtividade médias foram de 2523, 1463, 1397 e 2002 Mg.ha-1 para Água Branca, Arapiraca, Palmeira do Índios e Porto de Pedras.

\section{AGRADECIMENTOS}

Ao Instituto Nacional de meteorologia (INMET), ao Centro Nacional de Pesquisa de Solos UEP Recife da Empresa Brasileira de Pesquisa Agropecuária (EMBRAPA), e o Instituto Agronômico de Pernambuco - IPA e Fundação de Amparo à Pesquisa do Estado de Alagoas.

\section{REFERÊNCIAS}

ALLEN, R. G.; PEREIRA, L. S; RAES, D.; SMITH, M. Crop evapotranspiration guidelines for computing crop water requirements. (Irrigation and Drainage Paper, 56). Rome: FAO, 1998.

ALVES, M.E. B..; ANDRADE, C.L.T.; CÁRDENAS, R.R..; AMARAL, T.A.; SILVA, D.F. Identificação e quantificação do efeito de fatores ambientais na produtividade da cultura do milho na região de Janaúba, MG. Revista Brasileira de Agricultura Irrigada, v. 5, nº 3, p. 188 - 201, 2011. HTTP://DX.DOI.ORG/10.7127/RBAI.V5N300057

ANDRIOLI, K.G.; SENTELHAS, P.C. Brazilian maize genotypes sensitivity to water deficit estimated through a simple crop yield model. Pesquisa Agropecuária Brasileira, Brasília, v. 44, p. 653-660, 2009. http://dx.doi.org/10.1590/S0100-204X2009000700001

ASSAD, E.D.; MARIN, F.R.; EVANGELISTA, S.R.; PILAU, F.G.; FARIAS, J.R.B.; PINTO, H.S.; JÚNIOR, J.Z. Sistema de previsão de safra para o Brasil. Pesquisa Agropecuária Brasileira, Brasília, v. 42, p. 615-625, 2007 http://dx.doi.org/10.1590/S0100204X2007000500002

BARROS, H.C.B. Desenvolvimento de funções de pedotransferência e sua utilização em modelo agro-hidrológico. 2010. 148 p.Tese (Doutorado em Ciências). Escola Superior de Agricultura "Luiz de Queiroz". Universidade de São Paulo. Piracicaba, SP. 2010.

BONNECARRÈRE, R.A.G.; DOURADO-NETO, D.; MARTIN, T.N.; PEREIRA, A.R.; MANFRON, P.A. Estimativa das produtividades potencial e deplecionada da cultura do milho no Estado do Rio Grande do Sul em função das condições climáticas. Revista Brasileira de Agrometeorologia, Santa Maria, v. 15, p. 280-288, 2007.

CAMARGO, A.P.; SENTELHAS, P.C. Avaliação do desempenho de diferentes métodos de estimativa da evapotranspiração potencial no estado de São Paulo, Brasil. Revista Brasileira de Agrometeorologia, Santa Maria, v. 5, p. 89-97, 1997 
DE WIT, C.T. Photosynthesis of leaf canopies. Wageningen: PUDOC, 1965. 57 p.(Agriculture Research Report, 663).

DOORENBOS, J.; KASSAM, A.M. Yield response to water. Rome: FAO, 1979. 300 p. (Irrigation and Drainage Paper, 33).

EMBRAPA.

http://sistemasdeproducao.cnptia.embrapa.br/FontesHTML/Sorgo/CultivodoSorgo_4ed/cli ma.htm.Acesso em 04/12/2013.

INSTITUTO BRASILEIRO DE GEOGRAFIA E ESTATÍSTICA - IBGE. Disponível em: < http://www.sidra.ibge.gov.br/bda/agric/default.asp? $\mathrm{z}=\mathrm{t} \& \mathrm{o}=11 \& \mathrm{i}=\mathrm{P}>$. Acesso em: 15 dezembro de 2013.

MAGALHÃES, P. C.; DURÂES, F.O.M.; SHAFFERT, R.E. fisiologia da planta do sorgo. Embrapa milho e sorgo. Circular técnico, 3, 46p. 2000.

MARTINS, M. A. Estimativa de produtividade das culturas do milho e do sorgo a partir de Modelos Agrometeorológicos em algumas Localidades da Região Nordeste do Brasil. 2012. 127 p. Dissertação (Mestrado em Mestrado em Meteorologia). Instituto Nacional de Pesquisas Espaciais. São José dos Campos, SP.

OLIVEIRA, J.S.; FERREIRA, R.P.; CRUZ, C.D. et al. Adaptabilidade e estabilidade em cultivares de sorgo. Revista Brasileira de Zootecnia, v.31, n.2, p.883-889, 2002. http://dx.doi.org/10.1590/S1516-35982002000400011

ROLIM, G.S.; SENTELHAS, P.C.; UNGARO, M.R.G. Análise de risco climático para a cultura de girassol, em algumas localidades de São Paulo e do Paraná, usando os modelos DSSAT/OILCROP-SUN e FAO. Revista Brasileira de Agrometeorologia, Santa Maria, v. 9, p. 91-102, 2001.

SANTOS, R.D.; PEREIRA, L.G.R.; NEVES, A.L.A.; RODRIGUES, J.A.S.; COSTA, C.T.F.; OLIVEIRA, G.F. Agronomic characteristics of forage sorghum cultivars for silage production in the lower middle San Francisco Valley. Acta Scientiarum. Animal Sciences Journal. v. 35, n. 1, p. 13-19, Jan.-Mar., 2013.

SHRESTHA, N.; GEERTS, S.; RAES, D.; HOREMANS, S.; SOENTJENS, S.; MAUPAS, F.; CLOUET, P. Yield response of sugar beets to water stress under western European conditions. Agricultural Water Management, Amsterdam, v. 97, p. 346-350, 2010. http://dx.doi.org/10.1016/j.agwat.2009.10.005

TABOSA, J. N. Cultivo do Sorgo Granífero no Nordeste. In: Maria Elisa Ayres Guidetti Zagatto Paterniani; Aildson Pereira Duarte; Alfredo Tsunechiro. (Org.). Diversidade e Inovações na Cadeia Produtiva de Milho e Sorgo na era dos Transgênicos. 1ed. Campinas SP: Instituto Agronômico de Campinas / Associação Brasileira de Milho e Sorgo, 2012, v. 1, P. 581-604.

TABOSA, J. N.; SIMPLÍCIO, J.B.; NASCIMENTO, M. M. A. do; REIS, O. V. dos; SILVA, F. G.da; LIMA, J. M. P. Comportamento de Cultivares de Sorgo Forrageiro em Diferentes Ambientes do Semi-Árido Nordestino. In: XXVII CONGRESSO NACIONAL DE MILHO E SORGO, 2008, LONDRINA. ANIS CD- ROM DO XXVII CONGRESSO NACIONAL DE MUILHO E SORGO. LONDRINA: IAPAR / EMBRAPA, 2008. 


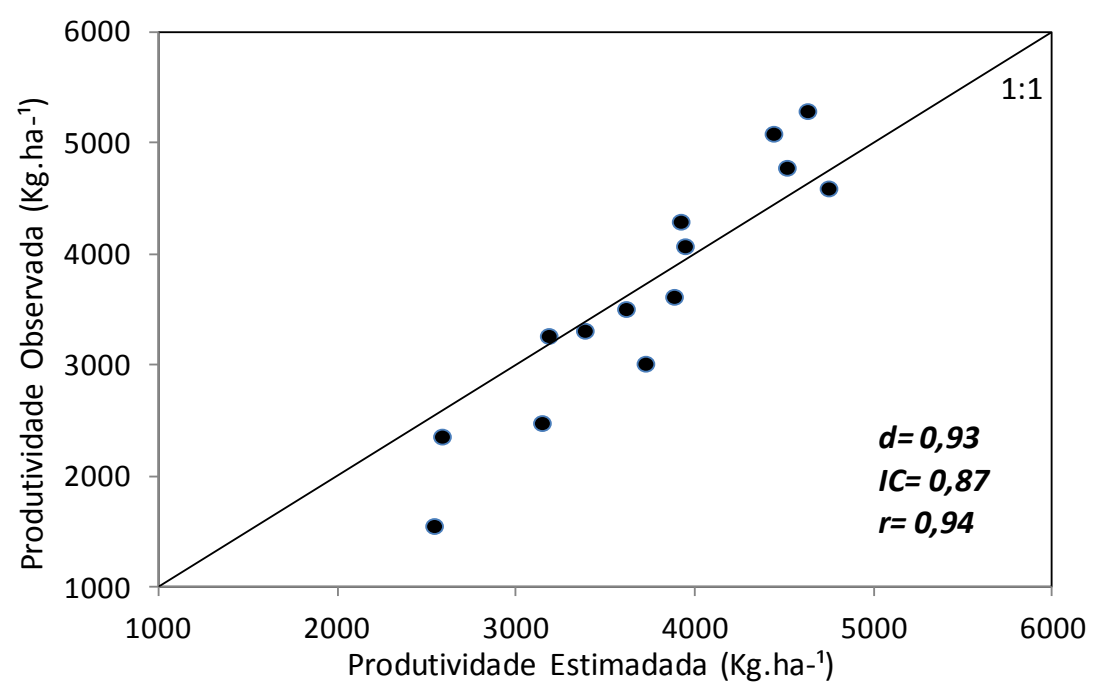

Figura 1. Relação entre produtividade observada e estimada com o modelo da Zona Agroecológica - FAO para cultura da sorgo.

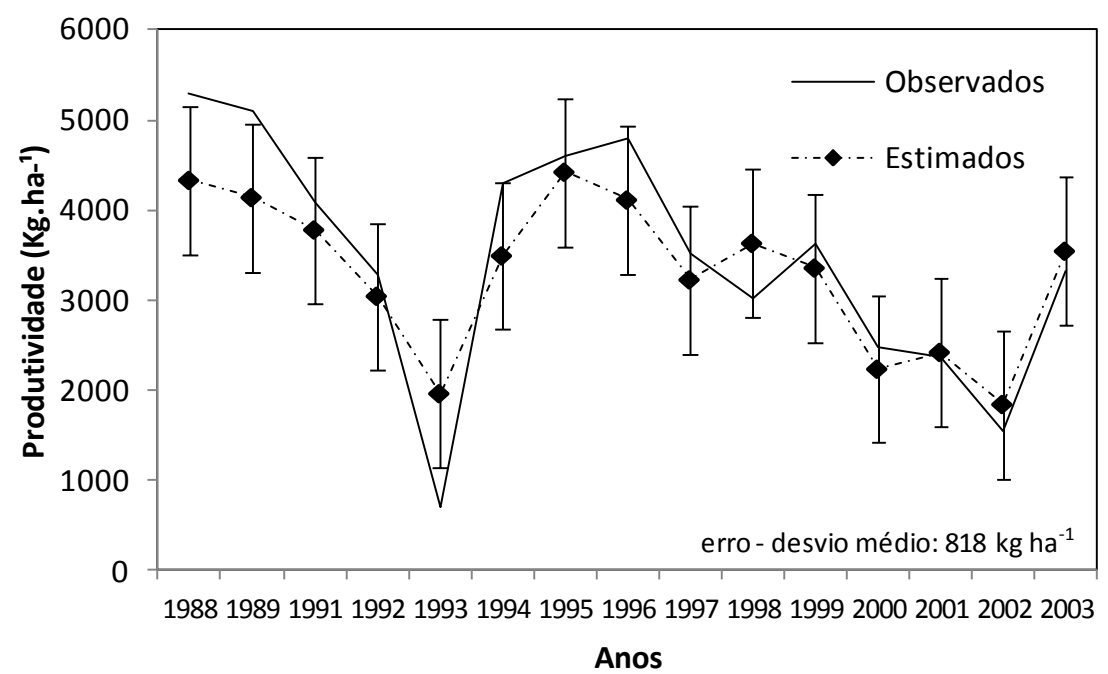

Figura 2. Erro médio entre produtividade observada e estimada com o modelo da Zona Agroecológica - FAO para cultura da sorgo.

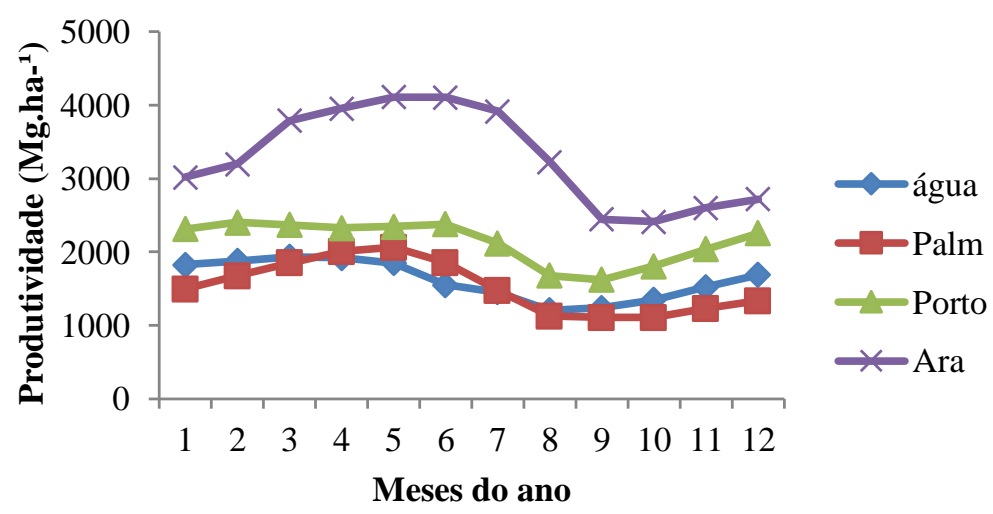

Figura 3. Produtividades em diferentes épocas do ano, para os municípios de Água Branca, Arapiraca, Palmeira do Índios e Porto de Pedras, em Alagoas, com CAD de 65 mm. 
Tabela 1. Produtividade da cultura do sorgo observada e estimada para localidade de Ararpina, PE.

\begin{tabular}{l|rcr}
\hline Ano & Observado & Estimado & \multicolumn{2}{c}{ Diferença } \\
\hline & \multicolumn{3}{|c}{ Produtividade $\left(\mathrm{Mg} \mathrm{ha}^{-1}\right)$} \\
1988 & 5297 & 4631 & 666 \\
1989 & 5093 & 4441 & 652 \\
1991 & 4079 & 3946 & 133 \\
1992 & 3270 & 3184 & 86 \\
1993 & 700 & 2471 & -1771 \\
1994 & 4300 & 3922 & 378 \\
1995 & 4600 & 4749 & -149 \\
1996 & 4786 & 4516 & 270 \\
1997 & 3513 & 3616 & -103 \\
1998 & 3020 & 3725 & -705 \\
1999 & 3623 & 3885 & -262 \\
2000 & 2485 & 3146 & -661 \\
2001 & 2363 & 2587 & -224 \\
2002 & 1557 & 2543 & -986 \\
2003 & 3317 & 3386 & -69 \\
Media & 3467 & 3650 & -183 \\
\hline
\end{tabular}

Tabela 2. Produtividades em diferentes épocas do ano, para os municípios de Água Branca, Arapiraca, Palmeira do Índios e Porto de Pedras, em Alagoas, para a CAD de $65 \mathrm{~mm}$, e redução percentual para a CAD $2(45 \mathrm{~mm})$ e $3(30 \mathrm{~mm})$.

\begin{tabular}{cccccccc}
\hline & \multicolumn{3}{c}{ Água Branca } & \multicolumn{3}{c}{ Arapiraca } \\
& CAD 1 & CAD 2 & CAD 3 & CAD 1 & CAD 2 & CAD 3 \\
& KG.ha-1 & $\%$ & $\%$ & KG.ha-1 & $\%$ & $\%$ \\
\hline jan & 1826.1 & -11.5 & -17.9 & 3017.2 & -11.6 & -17.7 \\
fev & 1876.9 & -8.4 & -13.6 & 3197.9 & -9.4 & -14.3 \\
mar & 1935.1 & -6.3 & -10.3 & 3789.0 & -5.7 & -9.7 \\
abr & 1921.5 & -5.9 & -9.7 & 3952.9 & -4.9 & -8.1 \\
mai & 1851.1 & -8.8 & -14.1 & 4108.8 & -5.1 & -9.0 \\
jun & 1551.9 & -12.6 & -19.2 & 4106.8 & -7.2 & -11.9 \\
jul & 1453.2 & -12.3 & -18.6 & 3915.5 & -9.1 & -15.1 \\
ago & 1205.8 & -16.1 & -23.8 & 3226.1 & -10.1 & -16.9 \\
set & 1236.5 & -16.3 & -24.1 & 2445.8 & -17.4 & -25.4 \\
out & 1348.2 & -15.6 & -23.3 & 2413.3 & -14.1 & -20.7 \\
nov & 1524.2 & -14.7 & -21.9 & 2601.8 & -14.4 & -22.0 \\
dez & 1689.1 & -13.4 & -20.5 & 2720.8 & -11.4 & -17.6 \\
\hline Média & 1618.3 & 1435.4 & 1337.0 & 3291.3 & 2983.1 & 1295.7 \\
Desvio & 268.1 & 292.0 & 296.3 & 659.4 & 717.4 & 384.5 \\
CV & 16.6 & 20.3 & 22.2 & 20.0 & 24.0 & 29.7 \\
\hline & Palmeira dos Índios & & Porto de Pedras \\
\cline { 2 - 4 } jan & 1493.9 & -11.6 & -17.1 & 2310.5 & -7.9 & -13.4 \\
& 14.9 & & & & &
\end{tabular}




\begin{tabular}{ccccccc} 
fev & 1673.6 & -9.5 & -14.3 & 2400.6 & -5.1 & -9.1 \\
mar & 1847.3 & -7.4 & -11.2 & 2368.4 & -2.7 & -4.9 \\
abr & 2002.7 & -4.7 & -7.7 & 2327.7 & -1.4 & -2.8 \\
mai & 2063.5 & -4.5 & -7.6 & 2346.6 & -1.5 & -2.9 \\
jun & 1853.2 & -8.7 & -13.6 & 2379.2 & -5.1 & -9.0 \\
jul & 1474.6 & -10.9 & -16.2 & 2118.7 & -9.1 & -13.7 \\
ago & 1125.8 & -13.2 & -19.6 & 1676.8 & -12.0 & -17.4 \\
set & 1103.7 & -16.0 & -23.2 & 1621.4 & -14.2 & -20.4 \\
out & 1103.7 & -16.0 & -23.2 & 1804.7 & -13.1 & -19.3 \\
nov & 1230.7 & -14.7 & -21.4 & 2038.3 & -12.3 & -18.6 \\
dez & 1333.4 & -13.3 & -19.3 & 2255.7 & -9.5 & -15.3 \\
\hline Média & 1525.5 & 1372.7 & 1295.7 & 2137.4 & 1980.3 & 1889.1 \\
Desvio & 355.9 & 380.4 & 384.5 & 286.7 & 346.0 & 363.5 \\
CV & 23.3 & 27.7 & 29.7 & 13.4 & 17.5 & 19.2 \\
\hline
\end{tabular}

